\title{
Percepción de los usuarios de la farmacia comunitaria sobre la COVID-19
}

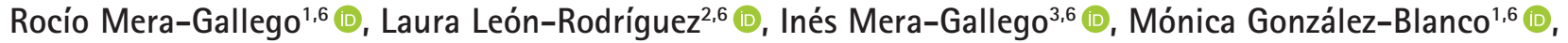

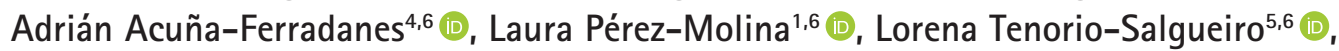 \\ José A. Fornos-Pérez ${ }^{5,6}$ (D) N. Floro Andrés-Rodríguez ${ }^{6}$ (i)
}

1. Farmacéutica comunitaria en Vigo. 2. Farmacéutica comunitaria en Ourense. 3. Farmacéutica comunitaria en Maella (Zaragoza). 4. Farmacéutico comunitario en Bueu (Pontevedra). 5. Farmacéutica comunitaria en Cangas do Morrazo (Pontevedra). 6. Grupo Berbés de Investigación y Docencia.

\section{PALABRAS CLAVE}

Farmacia comunitaria, SARS-CoV-2, COVID-19, percepción, estado de ánimo, vacunación

\section{ABREVIATURAS}

COVID-19: enfermedad infecciosa por coronavirus-19

OMS: Organización Mundial de la Salud

RD: Real Decreto

SARS-CoV-2: agente etiológico del sindrome agudo respiratorio severo por coronavirus-2 SS: sistema sanitario

\section{RESUMEN}

Objetivos: evaluar la percepción de los usuarios de las farmacias sobre la repercusión de la pandemia COVID-19 en su salud, el conocimiento sobre su posible inclusión en grupos de riesgo y su actitud ante una posible vacuna.

Material y métodos: estudio observacional transversal aleatorizado, en farmacias de Pontevedra y Ourense, desde marzo a junio de 2020 al comienzo y después de la restricción de movimientos.

Sujetos: usuarios $\geq 18$ años que acuden a las farmacias participantes en demanda de medicamentos y/o material de higiene y protección.

Procedimiento: el usuario cumplimentaba un cuestionario anónimo, depositándolo hasta la noche en una bandeja de plástico desinfectada diariamente. El procedimiento se repetirá tras cesar el confinamiento.

Resultados de la primera fase: se realizaron 706 encuestas. 415 (58,8 \%) mujeres, edad media 48,9 $(\mathrm{DE}=16,9)$ años. 100 participantes $(14,2 \%)$ viven solos.

$637(90,2 \%)$ creen que la COVID-19 es más peligrosa que la gripe, $189(26,8 \%)$ desconocen estar en algún grupo de riesgo.

$107(15,1 \%)$ dicen sentirse mal o muy mal con el aislamiento. Los aspectos más afectados son: familiar 350 (49,6\%) y emocional 338 (47,9\%).

En 2019/20 se vacunaron de la gripe $172(24,4 \%)$ y en 2020/21 piensan vacunarse $243(34,4 \%) .448$ (63,5 \%) se vacunarán frente a la COVID-19 cuando exista vacuna, esté o no financiada por el Sistema Nacional de Salud, 183 (25,9 \%) se lo pensarán.

Conclusiones: los encuestados consideran la COVID-19 más peligrosa que la gripe estacional. En esta primera fase la afectación sobre bienestar y salud no parece elevada. Un alto porcentaje se vacunará frente a la COVID-19.

\section{Introducción}

El 31 de diciembre de 2019 las autoridades sanitarias chinas informaron de un brote de 27 casos de neumonía, 7 de ellos graves, en la ciudad de Wuhan (provincia de Hubei, China) de etiología desconocida, con síntomas que se habían iniciado el 8 de diciembre de 2019. El 7 de enero de 2020, identificaron como agente causante a un virus de la familia Coronaviridae, denominado síndrome agudo respiratorio severo por coronavirus-2 (SARS-CoV-2).
La secuencia genética fue compartida por las autoridades chinas el 12 de enero (1).

El Comité de Emergencias del Reglamento Sanitario Internacional declaró el actual brote del nuevo coronavirus transformado en pandemia mundial como una Emergencia de Salud Pública de Importancia Internacional el 30 de enero de 2020 (2).

En España la mayor parte de las autoridades e instituciones de todos los ámbitos recomendaron o impusieron
Recibido: $16 / 6 / 2020$

Aceptado: 25/6/2020

Disponible online: $22 / 7 / 2020$
Financiación: ninguna.

Conflicto de intereses: ninguno.

Cite este artículo como: Mera-Gallego R, León-Rodriguez L, Mera-Gallego I, González-Blanco M, Acuña-Ferradanes A Pérez-Molina L, Tenorio-Salqueiro L, Fornos-Pérez JA, Andrés-Rodríquez NF. Percepción de los usuarios de la farmacia comunitaria sobre la COVID-19. Farmacéuticos Comunitarios. 2020 Jul 22;12(3):5-13. doi:10.33620/FC.2173-9218.(2020/ Vol12).003.02

Correspondencia: Rocio Mera-Gallego (rmeragallego@gmail.com).

ISSN 1885-8619 @ SEFAC (Sociedad Española de Farmacia Familiar y Comunitaria). Todos los derechos reservados. 
medidas de restricción o limitación de movimientos, de actividades comerciales, deportivas, sociales o viajes procedentes de lugares de riesgo hasta llegar a la declaración del estado de alarma el 15 de marzo de 2020 (3).

Esta nueva pandemia mundial denominada por la Organización Mundial de la salud (OMS) como COVID-19 (enfermedad infecciosa por coronavirus-19) está provocando un enorme impacto en la población. Por otro lado, las medidas de drástica restricción de la libertad de movimientos acordadas por el Gobierno español tras la declaración del estado de alarma, si bien pueden ser en algún grado necesarias con la finalidad de reducir la propagación del agente etiológico, suponen cambios importantes en el desarrollo normal de la vida privada, familiar, laboral y social, que se pueden ver reflejados en alteraciones de su salud y estado de ánimo, generando sentimientos de inseguridad, temor y desánimo (4).

La situación adquiere especial relevancia teniendo en cuenta que no existe certidumbre sobre su evolución ni sobre el posible fin de dicha situación excepcional, y el impacto psicológico sobre la sociedad es muy probable que produzca elevados niveles de estrés, ansiedad, miedo y soledad, tanto en los colectivos más expuestos como los profesionales sanitarios, como en los más vulnerables: mayores, enfermos crónicos, etc. (5-7).

Con el objetivo de conocer la percepción de los usuarios de las farmacias sobre la gravedad y repercusión que la pandemia está teniendo en su vida cotidiana y su salud y con la finalidad de diseñar posibles actuaciones futuras de apoyo a la población desde las farmacias comunitarias, de educación sanitaria sobre medidas de protección, concienciación sobre la eventual vacunación frente al coronavirus causante, etc., nos hemos planteado realizar el presente estudio.

\section{Objetivos \\ General}

Evaluar en dos momentos, al inicio y después de la situación de restricción de libertad de movimientos, la percepción de los usuarios de las farmacias sobre la repercusión de la pandemia COVID-19 en su estado de salud y su actitud ante una posible vacuna.

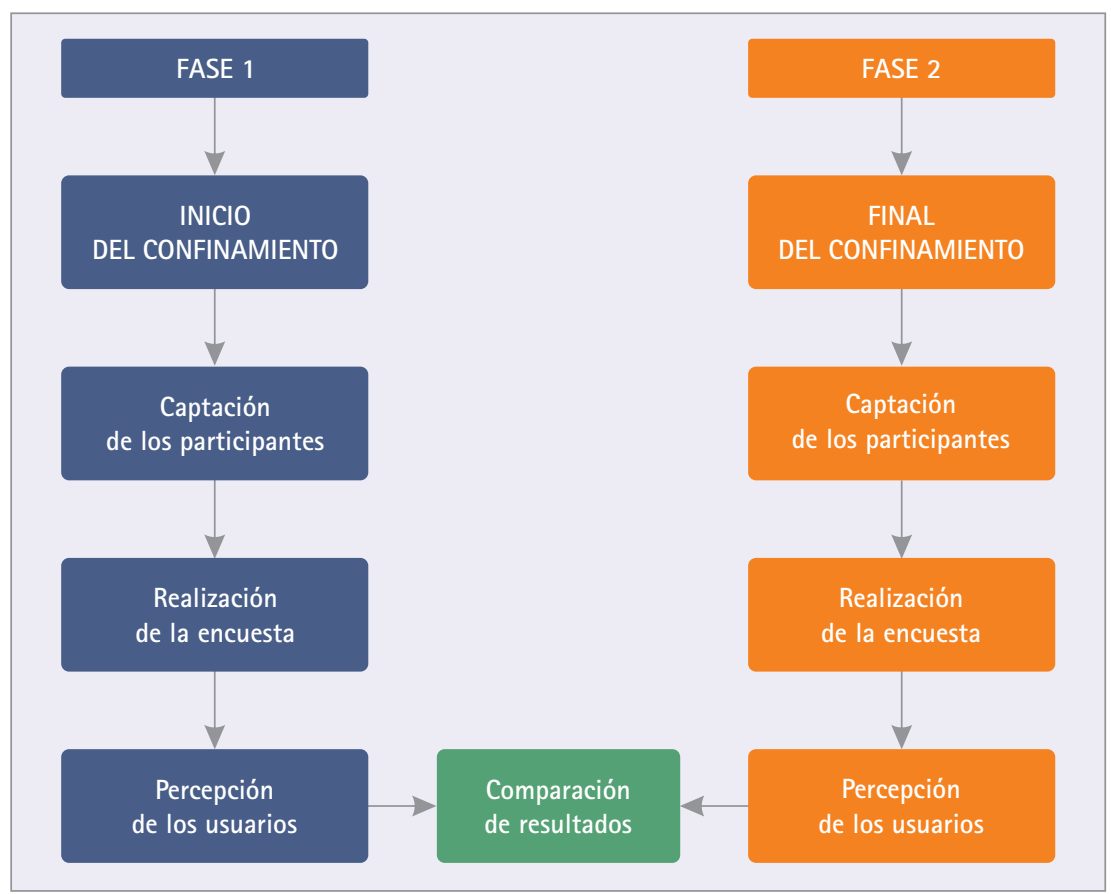

Figura 1 Esquema del estudio

\section{Específicos}

- Conocer su percepción sobre la peligrosidad del SARS-CoV-2 y el nivel de información que tienen sobre la posible pertenencia a grupos de riesgo.

- Valorar el grado de afectación que percibe sobre su salud a causa de las medidas de confinamiento domiciliario y restricción de movilidad.

- Averiguar su actitud ante la posibilidad a medio plazo de acceder a una vacuna frente a su agente etiológico.

- Comparar los resultados entre ambos momentos.

\section{Material y métodos}

\section{Diseño}

Estudio observacional transversal aleatorizado, realizado en farmacias de las provincias de Pontevedra y $\mathrm{Ou}$ rense, durante los meses de marzo a junio de 2020 en dos momentos: al inicio y después de la situación de restricción de libertad de movimientos.

\section{Sujetos}

Se incorporaron al estudio usuarios que acuden a las farmacias participantes en demanda de medicamentos, material de higiene y/o protección frente al SARS-CoV-2 Se ofreció participar a todos los usuarios mayores de 18 hasta alcanzar el número asignado a cada farmacia.

\section{Procedimiento (figura 1)}

Tras la explicación del objetivo del estudio y obtener su aceptación, se cumplimentaba el cuestionario, diseñado ad hoc por el grupo investigador (figura 2). Los realizados cada día se depositaban, una vez cumplimentados, hasta la noche en una bandeja de plástico, que se desinfectaba cada día. Los cuestionarios obtenidos se recogían al cierre de la farmacia con las mismas medidas de seguridad frente al contagio de la COVID-19 establecidas en los protocolos de actuación en las farmacias. Tras el levantamiento por parte del Gobierno de España de las restricciones de movilidad está previsto que se repita el mismo procedimiento descrito con una muestra equivalente con el fin de comprobar posibles cambios en la percepción de los participantes en ambas situaciones. En el presente trabajo se muestran los resultados de las encuestas realizadas al inicio de la situación de confinamiento, desde el 15 al 30 de marzo de 2020.

\section{Variables}

- Características demográficas del participante: edad (años), sexo $(\mathrm{M} / \mathrm{H})$, fumador (Sí/No), vive solo (Sí/No), trabaja durante el confinamiento (Sí/No).

- Valoración del usuario sobre la peligrosidad del SARS-CoV-2 y conocimiento de su pertenencia o no a un grupo de riesgo. 


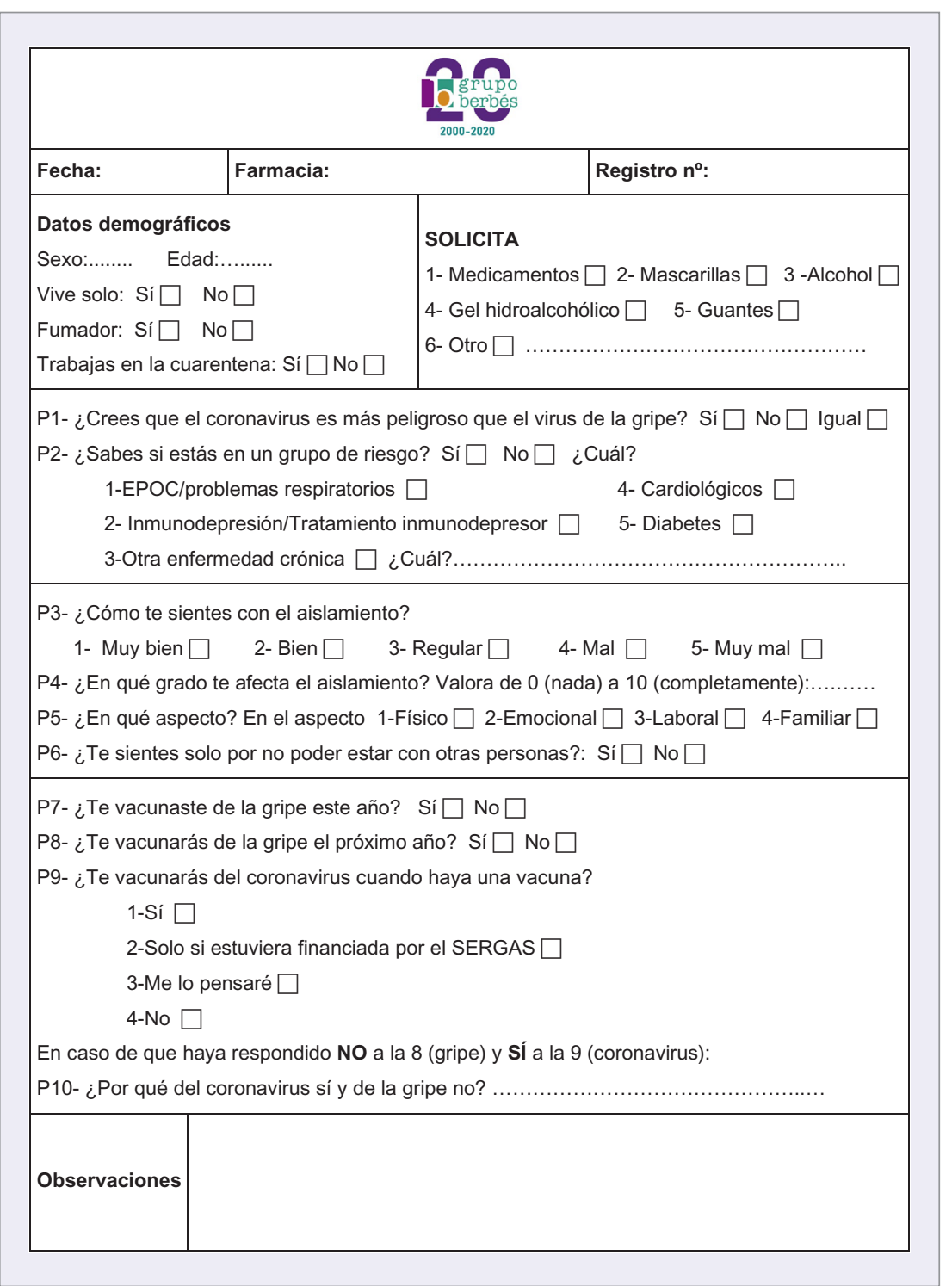

Figura 2 Cuestionario de percepción inicial sobre la pandemia de COVID-19

- Grado de afectación percibida sobre su salud a causa de las medidas de confinamiento (escala Likert de 5 posibilidades: muy bien/bien/regular/mal/muy mal, y escala continua de 0 a 10 puntos).

- Actitud ante la posible oferta a medio plazo de una vacuna contra el agente causante de la COVID-19 (Intención de vacunarse).

\section{Tamaño muestral}

Para conseguir una precisión del 5,0 \% en la estimación de una proporción mediante un intervalo de confianza asintótico Normal al $95 \%$ bilateral, asumiendo que la proporción es del 50,0 \%, resultó necesario incluir 384 unidades experimentales en el estudio. Previendo una proporción de un $10 \%$ de pérdidas, cuestionarios defectuosos o incompletos, analíticas de correlación de Spearman. La significación estadística se fijó en $\mathrm{p}<0,05$.

\section{Consideraciones éticas}

El estudio se ha desarrollado de acuerdo con las normas de Buena Práctica Clínica de la Conferencia Internacional sobre Armonización (ICH E6) para un estudio de estas características. Se tuvo en cuenta todos los requisitos legales aplicables $\mathrm{y}$, en particular, las leyes 41/2002 de 14 de noviembre, de autonomía del paciente, la ley 14/2007 de investigación biomédica, el Real Decreto (RD) 1720/2007 de 21 de diciembre, el RD 1716/2011, el RD 1090/2015, las normas de Buena Práctica Clínica (CPMP/ICH/135/95), el Reglamento (UE) $n^{\circ}$ 2016/679 General de Protección de Datos, etc.

\section{Confidencialidad}

de la información

En el propio cuestionario no se recogió ningún dato que permitiera la identificación de los participantes. El procedimiento garantiza que la cumplimentación y recogida de los cuestionarios y el análisis de los datos, características y opiniones de los participantes se realizó igualmente de manera totalmente anónima.

\section{Resultados}

Se presentan los resultados al inicio de la situación de restricción de movilidad. En el estudio participaron seis farmacias de Pontevedra y dos de Ourense, que realizaron 706 encuestas.

Presentación de los resultados y análisis estadístico

Para el análisis de los datos se ha utilizado el programa estadístico SPSS $^{\circledR} 22.0$ para Windows ${ }^{\circledR}$. Los datos cualitativos se expresaron como porcentajes y los cuantitativos como media \pm desviación estándar. Se utilizaron los test de chi-cuadrado para el análisis de variables cualitativas, t de Student para variables cuantitativas con distribución normal y Mann-Whitney para variables cuantitativas con distribución no normal. Para el análisis de los datos pareados se utilizó el test de Wilcoxon. Para relacionar las variables cuantitativas se utilizaron técnicas
Características de la muestra

Las características de los usuarios participantes se muestran en la tabla 1. Los porcentajes se refieren a la columna excepto en la distribución de la muestra por sexos (fila 1). $96(94,5 \%)$ personas encuestadas de 70 o más años solicitan medicamentos, frente a $453(73,7 \%)$ menores de 70 años, $\mathrm{p}<0,0001$. $26(31,9 \%)$ personas de 70 o más años solicitan guantes frente a 137 $(22,3 \%)$ menores de $70, p<0,05$. En el resto de items sobre la solicitud en la farmacia no hay diferencias significativas. 
Tabla 1 Características de los usuarios participantes

\begin{tabular}{|c|c|c|c|c|}
\hline & $\begin{array}{c}\text { Mujeres } \\
\text { n (\%) }\end{array}$ & $\begin{array}{c}\text { Hombres } \\
\text { n (\%) }\end{array}$ & $\begin{array}{l}\text { Total } \\
\text { n (\%) }\end{array}$ & P-valor \\
\hline Sexo & $415(58,8)$ & $291(41,2)$ & 706 (100) & \\
\hline Edad m (DE) (Rango) & $49,5(17,2)(18-90)$ & $48,2(16,4)(18-86)$ & $48,9(16,9)(18-90)$ & 0,3770 \\
\hline $\begin{array}{l}\text { Distribución etaria } \\
18-29 \\
30-39 \\
40-49 \\
50-59 \\
60-69 \\
70-79 \\
80-89 \\
\geq 90\end{array}$ & $\begin{array}{c}52(12,5) \\
88(21,2) \\
84(20,2) \\
57(13,7) \\
79(19,0) \\
35(8,4) \\
19(4,6) \\
1(0,2)\end{array}$ & $\begin{array}{c}36(12,4) \\
65(22,3) \\
60(20,6) \\
55(18,9) \\
39(13,4) \\
27(9,3) \\
9(3,1) \\
0(0,0)\end{array}$ & $\begin{array}{c}88(12,5) \\
153(21,7) \\
144(20,4) \\
112(15,9) \\
118(16,7) \\
62(8,7) \\
28(4,0) \\
1(0,1)\end{array}$ & \\
\hline Vive solo & $63(15,2)$ & $37(12,7)$ & $100(14,2)$ & 0,3550 \\
\hline Fumador & $61(14,7)$ & $53(18,2)$ & $114(16,2)$ & 0,2116 \\
\hline $\begin{array}{l}\text { Trabaja durante el confinamiento } \\
\text { Teletrabajo }\end{array}$ & $\begin{array}{l}131(31,6) \\
17(13,0)\end{array}$ & $\begin{array}{c}109(37,5) \\
8(7,3)\end{array}$ & $\begin{array}{l}240(34,0) \\
25(10,4)\end{array}$ & 0,1038 \\
\hline \multicolumn{5}{|l|}{ Solicita en la farmacia } \\
\hline Medicamentos & $327(78,8)$ & $212(72,9)$ & $539(76,3)$ & 0,0674 \\
\hline Mascarillas & $172(41,4)$ & $121(41,6)$ & $293(41,5)$ & 0,9714 \\
\hline Gel hidroalcohólico & $115(27,7)$ & $90(30,9)$ & $205(29,0)$ & 0,3540 \\
\hline Guantes & $103(24,8)$ & $63(21,6)$ & $166(23,5)$ & 0,3283 \\
\hline Consejo & $28(6,7)$ & $17(5,8)$ & $45(6,4)$ & 0,6280 \\
\hline Alcohol & $17(4,1)$ & $11(3,8)$ & $28(4,0))$ & 0,9598 \\
\hline Termómetro & $5(1,9)$ & $9(3,1)$ & $14(2,0)$ & 0,0765 \\
\hline Vitaminas & $8(1,2)$ & $2(0,7)$ & $10(1,4)$ & 0,1698 \\
\hline Otros & $33(8,0)$ & $31(10,7)$ & $64(9,1)$ & 0,7123 \\
\hline
\end{tabular}

Percepción sobre la enfermedad

637 encuestados (90,2 \%) creen que el SARS-CoV-2 es más peligroso que el virus de la gripe, $29(4,1 \%)$ que no y $40(5,7 \%)$ que es igual de peligroso. Esta percepción es mayor en las mujeres, 386 (93,0 \%), que en los hombres, $251(86,3 \%) \mathrm{p}<0,01$. No hay diferencia con la edad, si vive solo, si fuma, ni si trabaja, ni si se considera grupo de riesgo.

$189(26,8 \%)$ desconocen si están en algún grupo de riesgo, sin diferencias entre sexos. 77 (84,6 \%) usuarios de 70 o más años creen que son grupo de riesgo, frente a $440(71,5 \%)$ de menos de 70 años que también lo creen, $p<0,01$. Los grupos o factores de riesgo manifestados por los encuestados aparecen reflejados en la tabla 2.

\section{Estado anímico}

La media de las respuestas a la pregunta “¿Cómo te sientes con el aislamiento?" fue de 2,8 $(\mathrm{DE}=0,8)$ sobre $5,2,8(\mathrm{DE}=0,8)$ en las mujeres y $2,7(\mathrm{DE}=0,8)$ en los hombres. No hubo diferencias con respecto a la edad $(\mathrm{p}=0,7177)$.

En la tabla 3 se muestra la distribución de las respuestas según la escala Likert utilizada. No se encontraron diferencias en la gradación de la respuesta según el sexo, si vive solo, si fuma, si trabajan durante la cuarentena, ni si se consideran grupos de riesgo. Pero sí con la edad: $50(55,0 \%)$ de 70 o más años se sienten bien o muy bien con el aislamiento, frente a $229(37,2 \%)$ de los menores de 70 años, $p<0,01$.

A la pregunta “¿En qué grado te afecta el aislamiento?” la respuesta media fue de 5,7 (DE=2,1) sobre 10 . La respuesta fue mayor en las mujeres que en los hombres: 6,0 (DE=2,1) vs $5,4(\mathrm{DE}=2,1) \mathrm{p}<0,0001$. Resultó independiente de la edad.

$54(7,6 \%)$ encuestados, 37 (8,9\%) mujeres y 17 (5,8 \%) hombres, reconocieron una elevada afectación (9-10 de la escala) y 26 (3,7 \%), 12 (2,9\%) mujeres y 14 (4,8 \%) hombres dijeron no sentirse afectados (0-1 de la escala).

292 (41,4\%) encuestados, 195 $(47,0 \%)$ mujeres y 97 (33,3\%) hombres, dijeron encontrarse solos al no poder estar con otras personas. Más los de 70 o más años, 53 (58,2 \%) vs 239 (39,0 \%), $\mathrm{p}<0,001$.

Los aspectos del bienestar que percibieron afectados se reflejan en la tabla 4: a $28(30,1 \%)$ personas de 70 o más años les afecta menos en el 
Tabla 2 Factores de riesgo de sufrir complicaciones por la COVID-19

\begin{tabular}{|l|c|c|c|c|}
\hline \multicolumn{1}{|c|}{ Factores de riesgo } & $\begin{array}{c}\text { Mujeres } \\
\text { n (\%) }\end{array}$ & $\begin{array}{c}\text { Hombres } \\
\text { n (\%) }\end{array}$ & $\begin{array}{c}\text { Total } \\
\text { n (\%) }\end{array}$ & P-valor \\
\hline Ninguno & $68(16,4)$ & $47(16,2)$ & $115(16,3)$ & 0,9339 \\
\hline Edad $\geq 70$ años & $55(13,3)$ & $36(12,4)$ & $91(12,9)$ & 0,3498 \\
\hline Patología cardiovascular & $67(16,1)$ & $60(20,6)$ & $127(18,0)$ & 0,1276 \\
\hline EPOC/Asma/Problemas respiratorios & $54(13,0)$ & $34(11,7)$ & $88(12,5)$ & 0,5990 \\
\hline Diabetes mellitus & $17(4,1)$ & $20(6,9)$ & $37(5,2)$ & 0,1032 \\
\hline Psicopatologías & $15(3,6)$ & $9(3,1)$ & $24(3,4)$ & 0,7065 \\
\hline Inmunodepresión/Tratamiento inmunosupresor & $10(2,4)$ & $4(1,4)$ & $14(2,0)$ & 0,3315 \\
\hline Cáncer & $5(1,2)$ & $4(1,4)$ & $9(1,3)$ & 0,8967 \\
\hline Alergias & $3(0,7)$ & $2(0,7)$ & $5(0,7)$ & 0,9577 \\
\hline Otras & $10(2,4)$ & $5(1,7)$ & $15(2,1)$ & 0,4581 \\
\hline
\end{tabular}

Tabla 3 Respuestas a la pregunta "¿Cómo te sientes con el aislamiento?"

\begin{tabular}{|l|c|c|c|c|c|}
\hline & Muy bien & Bien & Regular & Mal & Muy mal \\
\hline Mujeres n (\%) & $5(1,2)$ & $152(36,6)$ & $194(46,7)$ & $47(11,3)$ & $17(4,1)$ \\
\hline Hombres n (\%) & $5(1,7)$ & $117(40,2)$ & $126(43,3)$ & $33(11,3)$ & $10(3,4)$ \\
\hline Total $n(\%)$ & $10(1,4)$ & $269(38,1)$ & $320(45,3)$ & $80(11,3)$ & $27(3,8)$ \\
\hline P-valor & \multicolumn{3}{|r|}{0,8201} & \\
\hline
\end{tabular}

aspecto físico, frente a $267(43,4 \%)$ de los menores de 70 años, $\mathrm{p}<0,05$; lo mismo ocurre en el aspecto emocional: 33 (36,3\%) vs 305 (49,6\%), $\mathrm{p}<0,05$; laboral $3 \quad(3,3 \%)$ vs 197 $(32,0 \%) \mathrm{p}<0,0001$. Les afecta más en el aspecto familiar 57 (62,6\%) vs 290 $(47,2 \%) \mathrm{p}<0,01$ y en la soledad: 53 $(58,2 \%)$ de los de 70 o más años se sienten solos frente a 239 (38,9\%) de los menores de 70 años, $\mathrm{p}<0,0001$.

\section{Vacunación}

Dijeron haberse vacunado de la gripe estacional en la campaña anterior (2019-2020) $172(24,4 \%)$ encuestados, 109 (26,3\%) mujeres y $63(21,6 \%)$ hombres. En mayor proporción los de 70 o más años: 63 $(69,2 \%)$ vs $109(17,7 \%)$ de menos de $70, p<0,0001$. También dijeron haberse vacunado $97(60,6 \%)$ usuarios de 65 o más años frente a $75(14,7 \%)$ de menos de 65 años, $\mathrm{p}<0,001$.

Tabla 4 Respuestas a la pregunta "¿En qué aspectos te afecta el confinamiento?"

\begin{tabular}{|l|c|c|c|c|}
\hline & Fisico & Emocional & Laboral & Familiar \\
\hline Mujeres n (\%) & $157(37,8)$ & $233(56,1)$ & $105(25,3)$ & $209(50,4)$ \\
\hline Hombres n (\%) & $138(47,4)$ & $105(36,1)$ & $95(32,6)$ & $138(47,4)$ \\
\hline Total n (\%) & $295(41,8)$ & $338(47,9)$ & $200(28,3)$ & $350(49,2)$ \\
\hline P-valor & 0,0110 & $<0,0001$ & 0,0330 & 0,4420 \\
\hline
\end{tabular}

En la próxima campaña tienen intención de vacunarse $243(34,4 \%)$, 149 (35,9\%) mujeres y 94 (32,3\%) hombres. Con mayor intención los de 70 o más años $71(78,0 \%)$ vs 172 $(28,0 \%) \mathrm{p}<0,001$. Al igual que los de 65 o más años $114(71,3 \%)$ vs 129 $(23,6 \%) \mathrm{p}<0,0001$.

Las respuestas a la pregunta “ ¿Te vacunarás de la COVID-19 cuando haya vacuna?" se presentan en la tabla 5.

De los 463 (60,9\%) participantes que no tienen pensado vacunarse de la gripe estacional en la próxima campaña, $132(28,5 \%)$ sí se vacunarán de la COVID-19 cuando esté disponible una vacuna, $103(22,2 \%)$ lo harían solo si fuera financiada por el sistema sanitario (SS), 159 (34,3\%) se lo pensarán y $69(14,9 \%)$ no se vacunarán tampoco del nuevo coronavirus. Las razones por los que los 235 (33,3\% del total) encuestados de los dos primeros grupos consideran más importante la vacunación frente a la COVID-19 son porque: "es más 
Tabla 5 Respuestas a la pregunta "¿Te vacunarás de la COVID-19 cuando haya vacuna?"

\begin{tabular}{|l|c|c|c|c|}
\hline & Si & $\begin{array}{c}\text { Solo si estuviera } \\
\text { financiada por el SS }\end{array}$ & Me lo pensaré & No \\
\hline Mujeres n (\%) & $174(41,9)$ & $98(23,6)$ & $109(26,3)$ & $34(8,2)$ \\
\hline Hombres n (\%) & $106(36,4)$ & $70(24,1)$ & $74(25,4)$ & $41(14,1)$ \\
\hline Total n (\%) & $280(39,7)$ & $168(23,8)$ & $183(25,9)$ & $75(10,6)$ \\
\hline P-valor & \multicolumn{4}{|c|}{0,0731} \\
\hline
\end{tabular}

peligroso", 124 (52,8\%); “por no contagiar a familiares $\mathrm{u}$ otras personas", $53(22,6 \%)$; "si lo recomienda la OMS, las autoridades sanitarias o su médico", 12 (5,1\%); "si fuese obligatoria", $11(4,7 \%)$; por otro motivo, $35(14,9 \%)$. Sin diferencias por la edad.

Dentro de las personas con riesgo, se observa que este hecho no influye en la decisión de vacunarse frente al SARS-CoV-2. El 41,1\% de los que lo consideran más peligroso afirman que se vacunarán. El 52,5 \% de los que se sienten muy mal piensa vacunarse, frente al 30,0\% de los que se sienten muy bien en el confinamiento, $p<0,001$. La decisión de vacunarse aumenta con el grado de afectación por el aislamiento: 0: 21,1 \%, 10: 65,6\%.

\section{Discusión}

\section{Limitaciones del estudio}

Una de las posibles limitaciones del estudio es que el tipo de personas encuestadas no corresponde a los usuarios habituales de la farmacia, ya que esta encuesta se realizó durante el estado de alarma donde se les pidió a las personas mayores y/o de riesgo quedarse en casa. Puede ser este el motivo de la baja edad media de los usuarios participantes (menor de 50 años), inferior a otros estudios realizados en el mismo ámbito $(8,9)$.

Dado que estamos ante un estudio realizado en una situación novedosa, otra posible limitación podría ser que existen pocas posibilidades de comparar con otros estudios que analicen las percepciones vividas en estos momentos. Además, dependiendo en qué fase se encuentre el sujeto encuestado, haber padecido la enfermedad, él o algún familiar o conocido cercano, puede modificar su opinión debido al miedo y la incertidumbre existentes. Es decir, la salud mental de la persona encuestada puede influir en su percepción sobre la enfermedad (10).

\section{Características de la muestra}

Los sujetos encuestados son mayoritariamente mujeres $(58,8 \%)$ con una media de edad de 48,9 años, inferior a las personas que habitualmente acuden a la farmacia $(8,9)$. No obstante, el 42,1\% de las personas encuestadas se encontraban entre $30 \mathrm{y}$ 49 años; este rango coincide con los hijos de las personas mayores que se encuentran en aislamiento, edad sin grandes patologías $\mathrm{y}$, por tanto, menor riesgo de sufrir complicaciones de la infección por SARS-CoV-2.

Como en otros estudios, la muestra se compone mayoritariamente de mujeres $(8,9)$. Son las que se responsabilizan de la salud familiar, vienen a la farmacia o se preocupan por tener mayor protección frente a la enfermedad (mascarillas, geles, etc.). Además, a pesar de que no es estadísticamente significativo, quizás por el tamaño de la muestra, su esperanza de vida es más prolongada (11) y, por tanto, la proporción de las que viven solas es mayor que la de los hombres (12). Tal como hemos visto en el estudio, 15,2 \% de las mujeres frente al 12,7 \% de los hombres que han venido a la farmacia. No obstante, la población estudiada no es una muestra representativa de la sociedad, en la que, según los datos del Instituto Nacional de Estadística de 2019 (12), el 72,3\% de las personas que viven solas son mujeres. En nuestro estudio, representarían el $8,9 \%$ del total y los hombres el $5,2 \%$ del total de los usuarios que vinieron a la farmacia.

Por otro lado, dentro de los productos más solicitados en el momento de la encuesta, en el confinamiento fueron los medicamentos $(76,3 \%)$, siendo mayor esta solicitud en los mayores de 70 años (94,5\%), entendiendo que utilizan mayor número de medicamentos en este rango de población. Entre todos los demás productos solicitados no hay dife- rencia entre sexo, pero llama especialmente la atención, la diferencia en edad en la solicitud de guantes, donde existe un porcentaje mayor en la población de 70 años o más. Tal vez debido a la falsa percepción de seguridad adicional que proporcionan.

\section{Percepción sobre la enfermedad}

Entendiendo la percepción de la enfermedad como la representación cognitiva que influye directamente en la respuesta emocional del paciente a su enfermedad y su comportamiento para hacer frente a esta (13), en el presente trabajo hemos pretendido estudiarla en diferentes aspectos. En todos los apartados analizados se han intentado recoger los sentimientos y emociones que una enfermedad como la COVID-19 genera en la población estudiada y que dan forma a su percepción sobre ella.

Resultó curioso encontrar entre las respuestas que, a pesar de tratarse de un estudio en las primeras semanas de la situación de alarma, ya menos del $10 \%$ de la población que acude a la farmacia cree que la COVID-19 es igual o menos peligrosa que la gripe, lo que puede explicarse precisamente por el ámbito donde se realiza, espacio sanitario al que acuden a buscar elementos de protección y salud. Esta percepción de peligrosidad relativa es alta ya en esta fase inicial, a pesar de que en nuestra comunidad la incidencia de la COVID-19 fue más leve, lo que podría indicar el alto grado de incertidumbre sobre la evolución posterior.

Cabe destacar que entre las variables que se asocian a esta pregunta como la edad, si es fumador, si trabaja o si vive solo, o si está en algún grupo de riesgo no aportan diferencias significativas en su respuesta. Solamente se encuentra diferencia en el sexo, siendo las mujeres (93,0\%) las que en mayor medida la consideran más grave.

En cuanto al conocimiento sobre su posible situación en algún grupo de riesgo en relación a la enfermedad $(14,15)$, llama la atención que, entre los mayores de 70 años, el $84,6 \%$ de los pacientes consideran estar dentro de un grupo de riesgo. De aquí concluimos que el 15,4\% de los encuestados mayores de 70 años desconocen que la edad per se es un factor de riesgo de la enfermedad, 
tal como atestigua el hecho de que más del $80 \%$ de los fallecidos eran personas con 70 o más años (16). Por otro lado, casi el $30 \%$ de nuestra muestra desconoce si está en algún grupo de riesgo, lo que nos muestra la importancia de realizar campañas sobre la enfermedad y su prevención desde la farmacia comunitaria, ya que fue el único establecimiento sanitario donde la población podía acudir libremente durante esta pandemia.

\section{Estado de ánimo}

El estado anímico se determinó a través de una escala numérica, siendo la respuesta más frecuente "regular" (puntuación de 2,8 sobre $5 \mathrm{DE}=0,8$ ), sin encontrar diferencias significativas excepto en la edad. El 55 \% de las personas mayores de 70 años decían sentirse bien o muy bien con el aislamiento, frente al $37,2 \%$ de los menores de 70 años. Esto puede deberse a que el confinamiento hizo que gran parte de la población activa dejase de trabajar, modificando la rutina de su día a día, mientras que las personas mayores de 70, al estar la mayoría ya jubilados, no se vieron tan afectados por esta situación.

Se preguntaba también en qué aspectos afectaba el confinamiento. En nuestro trabajo, el 41,4\% afirmaron haberse sentido solos, principalmente las mujeres y personas mayores de 70 años. La Organización Mundial de la Salud (OMS), en un informe del 26/03/20, consideraba que la soledad era uno de los problemas que surgirían con el confinamiento (17) y diversos estudios la reconocen como factor predictivo principal para las psicopatologías derivadas: depresión, ansiedad y estrés postraumático $(18,19)$. En el estudio de González-Sanguino et al. (19), realizado en España a través de las redes sociales, el $45 \%$ de los encuestados había sentido la falta de compañía algunas veces o a menudo en la semana posterior al 15 de marzo, informando también las mujeres de mayor sentimiento de soledad no deseada, lo que coincide notablemente con nuestro resultado. En cambio, no coincide en el grupo etario ya que la mayor percepción de soledad en este estudio (18) se presenta en el grupo de 18 a 39 años, quizás por el sesgo metodológico, reconocido por los autores, de ser este grupo etario el más participante en el estudio por ser el que más utiliza las redes sociales, y menor la participación de los de más edad en la encuesta.

Es posible, por tanto, que al pretender proteger del contagio a las personas mayores y a los sistemas de salud de la consecuente sobrecarga mediante el aislamiento social, puedan derivarse serios problemas físicos y psicológicos ocasionados por los sentimientos de soledad no deseada.

En cuanto a las variables analizadas, cabe destacar que en el sexo femenino fueron los componentes emocional y familiar los que más se vieron afectados en esta fase inicial del confinamiento. En cambio, los hombres lo fueron más en los otros aspectos estudiados, físico y laboral.

\section{Vacunación}

La campaña de vacunación antigripal se lleva a cabo todos los años entre los meses de octubre y diciembre, con la principal finalidad de minimizar el impacto de la infección de este virus en el número de infectados y en la gravedad de sus complicaciones. En Galicia se está consiguiendo aumentar la tasa de cobertura, pero muy lentamente, llegando solamente, en el año 2019/20, al 60,4 \% de la población gallega mayor de 65 años (20).

En el presente trabajo, se observa que únicamente el 24,4 \% de los encuestados manifiesta haberse vacunado durante la campaña del 2019/20. Este pequeño porcentaje puede deberse a que la vacunación de la gripe va dirigida y está financiada por el sistema sanitario solo en grupos de riesgo como personas mayores de 65 años, personas de cualquier edad que vivan en instituciones cerradas, embarazadas o con patologías de riesgo (20), por lo que dada la relativa baja media de edad de los participantes en nuestro estudio no era de esperar un porcentaje mayor.

No obstante, la intención de vacunarse de la gripe en la próxima campaña aumenta hasta el 34,4 \% del total de los encuestados. Este incremento puede asociarse a la situación de estrés y miedo generado por la no existencia de tratamiento efectivo o vacuna frente a la COVID-19 (10). Otro posible motivo de este aumento, puede deberse a que quieran prevenir otro tipo de infecciones virales con incidencia estacional del invierno ante posibles rebrotes de COVID-19 (14).
En las personas mayores de 65 años la intención de vacunarse en la siguiente campaña alcanza el 71,3\%, cifras que se acercan a la tasa recomendada por el Consejo Internacional del Sistema Nacional de Salud para la campaña de la gripe 2020-2021. Esperan alcanzar cuotas por encima del $75 \%$ de vacunación en mayores de 65 años, el $60 \%$ en embarazadas y personas en grupos de riesgo (21). Sería una buena noticia que efectivamente se consiguiera este aumento en la cobertura de la vacunación.

A pesar de que la mayoría de la muestra opina que es más peligroso la COVID-19 que la gripe (90,2\%), solamente el 63,5\% (de los cuales el 23,8\% estarían condicionados a la financiación del sistema sanitario) muestra intención de vacunarse, siendo las mujeres más receptivas a ello. Estos datos no coinciden con los de un estudio francés que presenta una aceptación de la vacuna de un 77,4 \%, siendo mayor este porcentaje en los hombres (22). Esta baja proporción en la intención de vacunación no nos sorprende, ya que en la última década Europa tiene los índices más altos de respuestas negativas en la percepción de la importancia de las vacunas, seguridad y eficacia (23). Asimismo, llama la atención, que el porcentaje de intención de vacunación frente a COVID-19 no sea mayor, pues está comprobado que el entusiasmo o, al menos, la actitud positiva ante una vacuna suele ser mayor durante $\mathrm{y}$ después de una situación de pandemia (24). Tal vez al haberse realizado el estudio al principio del estado de alarma, aún no era completa la percepción de su utilidad. De todas formas, con esta proporción de vacunación sería suficiente para conseguir con cierto éxito la inmunidad grupal, en la que se establece como tasa de inmunidad necesaria entre el 55 y 82 \% (25).

Por otro lado, en el extremo contrario, el escepticismo o la indecisión vacunal de nuestro estudio es del $10,6 \%$ de la muestra, frente al $26 \%$ de la muestra francesa (22) y el $9 \%$ en los Estados Unidos (26). Un posible motivo, tanto en el presente estudio como en el realizado en los Estados Unidos, podría ser la existencia en ambos de la opción de respuesta "me lo pensaré”, ausente en el estudio francés, cuyos resultados corresponden al 25,9 \% y $15 \%$ respectivamente de las respuestas (26). 
Este escepticismo puede llegar a constituir la primera y principal barrera para implantar con éxito una futura campaña de vacunación frente al virus SARS-CoV-2 en el momento que esté disponible la vacuna. Por tanto, el farmacéutico y la farmacia comunitaria tendrán un papel fundamental en ella, participando en la educación sanitaria sobre la enfermedad y la vacuna, como ya viene realizando en otras infecciones en cuanto a información al paciente de la patología y animando su aceptación de la vacunación $(23,27)$. Pero, sobre todo, mediante una implicación más activa, que como farmacéuticos $\mathrm{y}$ pacientes reivindicamos (23), colaborando de manera efectiva en el proceso de administración, lo que se ha demostrado altamente eficiente en la consecución de altas tasas de inmunización (28).

\section{Conclusiones}

Los encuestados consideran la COVID-19 más peligrosa que la gripe estacional, pero más de la cuarta parte desconoce si está en algún grupo de riesgo. Las mujeres manifiestan una mayor percepción de peligro.

En esta primera fase la repercusión sobre bienestar y salud no parece ser de momento elevada. Los mayores de 70 años sufren menos impacto en su estado de ánimo, aunque se ven más afectados por la soledad, y en mayor medida las mujeres que los hombres.

Aumenta en más de un $10 \%$ la intención de vacunarse de la gripe, superando el $70 \%$ en los mayores de 65 años. Un porcentaje considerable, pero menor del esperado dada la situación, próximo al $65 \%$, se vacunará frente a la COVID-19 cuando esté disponible la vacuna.

\section{Agradecimientos}

A todos los usuarios de las farmacias que se prestaron amablemente a responder a nuestra encuesta y a los farmacéuticos comunitarios que desempeñan su labor en las farmacias que colaboraron en el estudio.

\section{Referencias bibliográficas}

1. Ministerio de Sanidad. Enfermedad por nuevo coronavirus, COVID-19. Información inicial de la Alerta en China. 31/1/2020. [Acceso 12/6/2020]. https://www.mscbs.gob.es/en/profesionales/saludPublica/ccayes/alertasActual/nCov-China/documentos/ Informacion_inicial_alerta.pdf

2. Organización Mundial de la Salud. Declaración sobre la segunda reunión del Comité de Emergencias del Reglamento Sanitario Internacional (2005) acerca del brote del nuevo coronavirus (2019-nCoV). [Acceso 12/6/2020]. Disponible en: https://www.who.int/ es/news-room/detail/30-01-2020-statement-on-the-second-meeting-of-the-international-health-regulations-(2005)-emergency-committee-regarding-the-outbreak-of-novel-coronavirus-(2019-ncov)

3. Gobierno de España. Real Decreto 463/2020, de 14 de marzo, por el que se declara el estado de alarma para la gestión de la situación de crisis sanitaria ocasionada por el COVID-19. «BOE" núm. 67, de 14/03/2020. https://www. boe.es/eli/es/rd/2020/03/14/463/con

4. EAE Bussines School. La crisis del coronavirus, la información y los estados anímicos. 1/4/2020. [Acceso 12/6/2020]. Disponible en: https://www.eae.es/ actualidad/noticias/la-crisis-del-coronavirus-la-informacion-y-los-estados-animicos

5. Ibáñez-Vizoso JE, Alberdi-Páramo I, Díaz-Marsá M, Perspectivas Internacionales en Salud Mental ante la pandemia por el nuevo coronavirus SARS-CoV-2. Revista de psiquiatría y salud mental (Barcelona). 2020. doi:10.1016/j.rpsm.2020.04.002

6. Cash R, Patel V. Has COVID-19 subverted global health? Lancet. 2020; 395:16871688. https://www.ncbi.nlm.nih.gov/ pmc/articles/PMC72001 22/

7. Ricci Cabello I, Meneses Echávez JF, Serrano-Ripoll MJ, Fraile-Navarro D, Fiol de Roque MA, Pastor Moreno G, et al. Impact of viral epidemic outbreaks on mental health of healthcare workers: a rapid systematic review. medRxiv preprint 2020.04.02.20048892. doi: doi:10.1101/2020.04.02.20048892

8. Fornos-Pérez JA, Andrés-Rodríguez NF, Andrés-Iglesias JC, Mera-Gallego R, Mera-Gallego I, Penín-Álvarez 0 , et al. Valoración del cumplimiento de los tratamientos hipoglucemiantes y antihipertensivos en Galicia ("CumpleGa"). Farmacéuticos Comunitarios 2017; 9(4):7-15. doi:10.5672/ FC.2173-9218.(2017/Vol9).004.02

9. Piñeiro-Abad A, Mera-Gallego R, Andrés-Iglesias JC, Fornos-Pérez JA, Vérez-Cotelo N, Andrés-Rodríguez NF. Análisis del rechazo de dispensaciones en receta electrónica y la relación con el incumplimiento. Pharm Care Esp. 2018; 20(4):247-268. https:// www.pharmcareesp.com/index.php/ PharmaCARE/article/view/441

10. Lozano-Vargas A. Impacto de la epidemia del Coronavirus (Covid-19) en la salud mental del persona de salud y en la población general de China. Rev Neuropsiquiatr. 2020; 83 (1):5156. doi:10.20453/rnp.v83i1.3687

11. Instituto Nacional de Estadística (INE). Salud. 4.- Esperanza de vida. Actualizado 5/6/2020. [Acceso 25/6/2020]. Disponible en: https://www.ine.es/ ss/Satellite?L=es_ESCtc=INESeccion_ CEtcid $=1259926380048 \mathrm{Ct} p=12547351$ 10672Etpagename=ProductosYServicios/PYSLayout

12. Instituto Nacional de Estadística (INE). Encuesta continua de hogares. Personas que viven solas. 2019. [Acceso 29/5/2020]. Disponible en: https:// www.ine.es/prensa/ech_2019.pdf

13. Quiceno J, Vinaccia S. Percepción de enfermedad: una aproximación a partir del Illness Perception Questionnaire. Psicología desde el Caribe 2010; 26:5683. https://www.redalyc.org/articulo.oa ?id=21315106004

14. Ministerio de Sanidad. Información Científica-Técnica. Enfermedad por coronavirus, COVID-19. Actualizado 2/6/2020. [Acceso 13/6/2020]. Disponible en: https://www.mscbs.gob.es/ en/profesionales/saludPublica/ccayes/ alertasActual/nCov-China/documentos/ITCoronavirus.pdf

15. Ministerio de Sanidad. Procedimiento de actuación para los servicios de prevención de riesgos laborales frente a la exposición al SARS-CoV-2. 8/6/2020. [Acceso 13/6/2020]. Disponible en: https://www.mscbs.gob. es/en/profesionales/saludPublica/ccayes/alertasActual/nCov-China/documentos/PrevencionRRLL_COVID-19. pdf

16. Ministerio de Sanidad. Datos de fallecidos por COVID-19 por grupos de población. [Acceso 10/6/2020]. Disponible en: https://www.mscbs.gob.es/profesionales/saludPublica/ccayes/alertasActual/ nCov-China/situacionActual.htm

17. OMS. Impacto psicológico de la $\mathrm{CO}$ VID-19. [Acceso 10/6/2020]. Disponible en https://www.dw.com/es/oms-el-impacto-psicol\%C3\%B3gico-del-covid-19-en-la-sociedad-no-debe-ser-ignorado/a-52925095

18. Brooks SK, Webster RK, Smith LE, Woodland L, Wessely S, Greenberg $\mathrm{N}$, et al. The psychological impact of quarantine and how to reduce it: rapid review of the evidence. Lancet 2020; 395:912-920, 10.1016/S01406736(20)30460-8. https://www.thelancet.com/journals/lancet/article/ PIIS0140-6736(20)30460-8/fulltext

19. González-Sanguino C, Ausín B, Castellanos MÁ, Saiz J, López-Gómez A, Ugidos C, et al. Mental health consequences during the initial stage of the 2020 coronavirus pandemic (COVID-19) in Spain. Brain, Behavior, and Immunity. 2020. doi:10.1016/j. bbi.2020.05.040

20. Xunta de Galicia. Dirección Xeral de Saúde Pública. Campaña de vacunación antigripal 2019. [Acceso 
9/6/2020]. Disponible en: https:// www.sergas.es/Saude-publica/Campa\%C3\%B1a-actual?idioma=es

21. Ministerio de Sanidad. Vacunación frente a la gripe. Grupos de población a vacunar. Temporada 2020-2021 [Acceso 13/6/2020]. Disponible en: https://www.mscbs.gob.es/profesionales/saludPublica/prevPromocion/ vacunaciones/docs/Recomendaciones_vacunacion_gripe.pdf

22. Detoc M, Bruel S, Frappe P, Botelho-Nevers $\mathrm{E}$, Gagneux-Brunon A. Intention to participate in a COVID-19 vaccine clinical trial and to get vaccinated against $\mathrm{CO}$ VID-19 in France during the pandemic. medRxiv 2020.04.23. 20076513; doi:10. 1101/2020.04.23.20076513

23. Andrés-Rodríguez NF, Mera-Gallego R, Piñeiro-Abad A, Acuña-Ferradanes
A, Mera-Gallego I, García-Rodríguez $\mathrm{P}$, et al. Vacunación antigripal en la farmacia comunitaria: opinión de pacientes y farmacéuticos. Farmacéuticos Comunitarios. 2018 Sep 28; 10(3):15-24. doi:10.5672/FC.21739218.(2018/Vol10).003.03

24. Chen RT, Orenstein WA. Epidemiologic methods in immunization programs. Epidemiol Rev. 1996;18(2):99-117. doi:10.1093/oxfordjournals.epirev. a017931

25. Schaffer De Roo S, Pudalov NJ, Fu LY. Planning for a COVID-19 Vaccination Program. JAMA. 2020. https://jamanetwork.com/journals/jama/fullarticle/2766370

26. NBC4. Morning Consult. National Tracking Poll \#200395 March 2425, 2020. Encuesta estratificada so- bre vacunación frente a COVID-19. [Acceso 6/6/2020]. Disponible en: https://www.nbcwashington.com/ wp-content/uploads/2019/09/LX_COVID_Vaccine_Poll_crosstabs.pdf

17. Aparicio C, Climent MT, Baixauli VJ, Rodrigo MJ, Albanell F, Recio MC. Intervención del farmacéutico comunitario en la vacunación de adultos con patología respiratoria. Farmacéuticos Comunitarios. 2018; 10(3):5-14. doi:10.5672/ FC.2173-9218.(2018/Vol10).003.02

28. Isenor JE, Alia TA, Killen JL, Billard BA, Halperin BA, Slayter KL, et al. Impact of pharmacists as immunizers on influenza vaccination coverage in Nova Scotia, Canada. Human Vaccines \&t Immunotherapeutics 2016; 12(5):1225-1228. doi:10.1080/216455 15.2015 .1127490 